\title{
Fluorescent Diarylethene Photoswitches-A Universal Tool for Super-Resolution Microscopy in Nanostructured Materials
}

\author{
Oleksii Nevskyi, Dmytro Sysoiev, Jes Dreier, Simon Christoph Stein, Alex Oppermann, \\ Florian Lemken, Tobias Janke, Jörg Enderlein, Ilaria Testa, Thomas Huhn, \\ and Dominik Wöll**
}

Super-resolution fluorescence microscopy allows for unprecedented in situ visualization of biological structures, but its application to materials science has so far been comparatively limited. One of the main reasons is the lack of powerful dyes that allow for labeling and photoswitching in materials science systems. In this study it is shown that appropriate substitution of diarylethenes bearing a fluorescent closed and dark open form paves the way for imaging nanostructured materials with three of the most popular super-resolution fluorescence microscopy methods that are based on different concepts to achieve imaging beyond the diffraction limit of light. The key to obtain optimal resolution lies in a proper control over the photochemistry of the photoswitches and its adaption to the system to be imaged. It is hoped that the present work will provide researchers with a guide to choose the best photoswitch derivative for super-resolution microscopy in materials science, just like the correct choice of a Swiss Army Knife's tool is essential to fulfill a given task.

of the relationship between structure, properties, and functions, a direct visualization of the underlying structures on the nanoscale is essential. Imaging methods, such as scanning electron microscopy (SEM), transmission electron microscopy (TEM), ${ }^{[6]}$ atomic force microscopy (AFM), ${ }^{[7]}$ and scanning tunneling microscopy (STM), often give useful insights, but have their limitations: they are either invasive, possess poor contrast, or need high-vacuum conditions. As a result, the sample preparation is complicated and time-consuming, which makes them hardly applicable for the study of dynamic processes in situ. Fluorescence microscopy (FM) techniques are noninvasive and allow for real-time imaging of structures that are specifically labeled with appropriate fluorophores.

\section{Introduction}

Nanoscopic structures are one of the keys of functional polymeric materials. ${ }^{[1-5]}$ In order to gain a detailed understanding

O. Nevskyi, A. Oppermann, F. Lemken, T. Janke, Prof. D. Wöll Institute for Physical Chemistry

RWTH Aachen University

Landoltweg 2, 52074 Aachen, Germany

E-mail:woell@pc.rwth-aachen.de

Dr. D. Sysoiev, Dr. T. Huhn

Department of Chemistry

University of Konstanz

Universitätsstr. 10, 78464 Konstanz, Germany

Dr. J. Dreier, Prof. I. Testa

Science for Life Laboratory

KTH Royal Institute of Technology

Tomtebodavägen 23A, 17121 Solna, Sweden

Dr. S. C. Stein, Prof. J. Enderlein

III. Institute of Physics - Biophysics

Georg-August University

Friedrich-Hund-Platz 1, 37077 Göttingen, Germany
However, classical FM provides a spatial resolution in the range of only $200-300 \mathrm{~nm}$, which is not sufficient to resolve most polymer structures which are rather on the 10-100 nm scale.

Modern FM methods circumvent the diffraction limit of classical microscopy, thus allowing for in situ imaging with a resolution on the nanoscale. Such super-resolution fluorescence microscopy methods can be categorized into deterministic methods, such as stimulated emission depletion (STED) microscopy ${ }^{[8]}$ and reversible saturable optical fluorescence transitions (RESOLFT) microscopy, ${ }^{[9]}$ into stochastic methods, such as (direct) stochastic optical reconstruction microscopy ((d)STORM) ${ }^{[10,11]}$ and photoactivated localization microscopy (PALM), ${ }^{[12]}$ and finally into image-correlation methods such as super-resolution optical fluctuation imaging (SOFI). ${ }^{[13]}$ Moreover, combinations of different super-resolution methods can give access to complementary information. ${ }^{[14]}$ Together with modern optics, the success of these nanoimaging approaches relies on a sophisticated control of the switching between dark and emissive states of fluorescent dyes or proteins for significantly enhancing the spatial resolution. ${ }^{[15-19]}$

As a result, numerous new possibilities have emerged in the life sciences involving the study of cell dynamics and the 
resolution of cell structures beyond the diffraction limit. For instance, using reversibly photoswitchable fluorescent proteins like rsEGFP, which undergo thousands of E/Z-isomerizations, $30 \mathrm{~nm}$ resolution could be reached under RESOLFT conditions. ${ }^{[20,21]}$ Synthetic organic fluorophores, unlike fluorescent proteins, are far less frequently used in RESOLFT microscopy. So far, there are only few examples of their successful applications described in the literature. ${ }^{[22,23]}$

Applying image-correlation methods such as SOFI open new possibilities for in situ dynamic investigations. Potentially capable of performing super-resolution imaging (SRI) at high frame rates, SOFI offers great opportunities for fluorescence nanoimaging. A significant advantage of SOFI is its robustness with respect to blinking modalities, which allows its application to various fluctuating samples such as rotating dipoles or binary stars, to be imaged and super-resolved. ${ }^{[13,24]}$

The visualization, with the above described methods, of apolar structures found in material science is often impossible since the employed polar switching buffers cannot significantly access apolar structures, whereas fluorescent proteins, if appropriate to be used, would denature under such conditions. As a consequence, super-resolution microscopy has so far not been used extensively to address topics in soft matter or polymer science. ${ }^{[25-35]}$

Universal application of SRI in the field of material science mostly depends on the availability of switchable dyes with suitable photophysical and chemical properties such as high fluorescence quantum yield, large separation between the absorption bands of the on- and off-state, high photostability, adjustable switching kinetics, minimum interaction with the environment, and a straightforward derivatization approach for specific structure labeling.

Diarylethenes (DAE) are one of the most promising classes of organic photoswitches, due to their various potential applications in data storage, chemical sensing, and as nanomaterials. ${ }^{[36-39]}$ Despite the multitude of different diarylethene derivatives available, only few of them exhibit, in their closed form, strong fluorescence in the visible range in conjunction with adjustable switching rates. As a consequence, their application for SRI has been limited so far. ${ }^{[26,40,41]}$

One way to address this challenge was to covalently link a diarylethene switch to a suitable fluorophore such as a perylene diimide, ${ }^{[42]}$ for utilizing the energy or electron transfer of the resulting multichromophoric system. The synthesis of such a dyad comprises several steps, with the rather large probe molecule altering the labeled structure, and might thus complicate the interpretation of the photochemistry and photophysics of the entire system.

Here, we present novel photochromic fluorescent diarylethene photoswitches with high photostability and strong fluorescence in their closed bathochromic forms. We show that these photoswitches can be readily used for different types of super-resolution microscopy in soft matter and polymer materials. In contrast to a previous communication where we have shown their applicability for PALM imaging, ${ }^{[26]}$ we demonstrate in this paper, how the photoswitching characteristics of the different derivatives can be exploited for different super-resolved fluorescence imaging techniques (PALM, RESOLFT, and SOFI). Thus, diarylethene derivatives are comparable to a Swiss Army Knife for SRI in materials science, allowing to address different questions by choosing the appropriate dye depending on the required temporal and spatial resolution, probe environment, and available equipment. We demonstrate this concept by imaging self-assembled block copolymers as a prominent example for soft matter structures. Additionally, depending on the research question and the technical equipment available, the possibility of one-color control of activation/deactivation of $\mathrm{DAE}^{[41]}$ can be achieved by an appropriate choice of one of the diarylethene derivatives reported here.

\section{Results and Discussion}

Different derivatives of sulfone based DAE (see Figure 1) were synthesized by Suzuki-Miyaura coupling reactions using the 6,6'-diiodo derivative of 1,2-bis(2-ethyl-1,1-dioxidobenzothiophene-3-yl)perfluorocyclopentene and the corresponding boronic acids (see the Supporting Information). ${ }^{[43]}$ Upon irradiation with UV light, the nonfluorescent open-ring form 1o-10o undergoes a cyclization reaction to yield the fluorescent closedring isomer 1c-10c. Irradiation with visible light results in opening of the closed-ring to the open-ring isomer. This photoswitching behavior is well-suited for SRI.

\subsection{Spectral Properties}

The structures of our diarylethene photoswitches and representative fluorescence spectra in the closed forms are presented in Figure 1. Compounds $10 \mathrm{c}-9 \mathrm{oc}$ are symmetric and contain substituents with strong electron donating properties (compounds $20 \mathrm{c}$ and $50 \mathrm{c}$ ) and with strong electron withdrawing properties (compounds $60 \mathrm{c}$ and $7 \mathrm{oc}$ ), respectively. Compound $10 \mathrm{oc}$ is an example of a push-pull dye combining an electron donating 4-methoxyphenyl fragment and an electron withdrawing 3,5-bistrifluoromethylphenyl group. This results in a bathochromic shift of the closed-ring isomer 10c absorption band in comparison to the closed ring isomer of the corresponding symmetric compounds. All the photophysical properties of the compounds 1 oc-10oc are summarized in Table S1 of the Supporting Information.

The closed ring isomers exhibit strong fluorescence in apolar surrounding with fluorescence quantum yields up to 0.84 in the wavelength range for the fluorescence band maxima between 510 and $585 \mathrm{~nm}$, as determined in 1,4-dioxane solution (see Figure 2 and Table S1, Supporting Information). All derivatives exhibit reasonable high fluorescence quantum yields in apolar media suitable for super-resolution microscopy. Compound 8oc shows a significant drop of the fluorescence quantum yield, presumably due to a nonplanar geometry in the excited state. In more polar solvents, the quantum yield of all derivatives drops significantly, and a bathochromic shift of the fluorescence of the closed form is observed (see Figure S2 and Table S2 in the Supporting Information). The fluorescence quantum yield does not significantly depend on the substituent, while the wavelength range of fluorescence can be tuned by proper substitution 

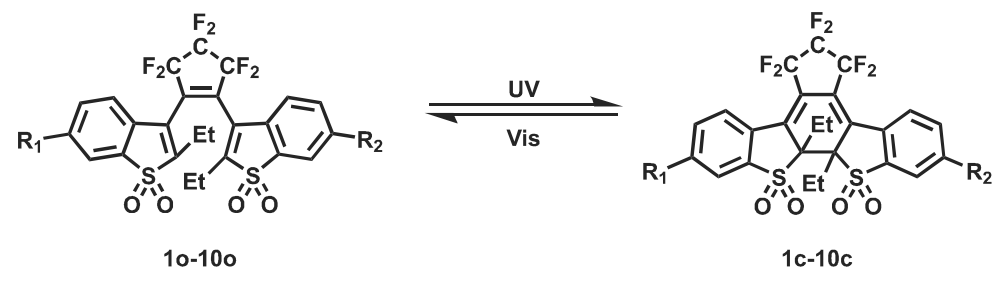<smiles></smiles>

$10 \mathrm{c}$<smiles>c1ccc(CC2CC2CC2CCCCC2)cc1</smiles>

$40 c$<smiles>Cc1cc(C)cc(C(F)(F)F)c1</smiles>

$7 \circ \mathrm{c}$<smiles>CCc1ccc(OC)c(OC)c1</smiles>

$20 c$<smiles>CCc1ccc(OC)cc1</smiles>

$50 \mathrm{c}$

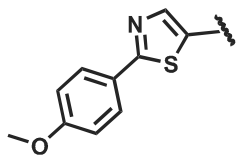

$80 c$

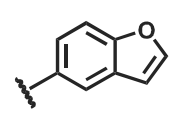

3oc<smiles>CCc1cccc(C(F)(F)F)c1</smiles>

$60 \mathrm{c}$

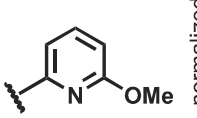

9oc<smiles>[R7]Cc1cc(CC)cc(C(F)(F)F)c1</smiles>

$10 \mathrm{oc}$

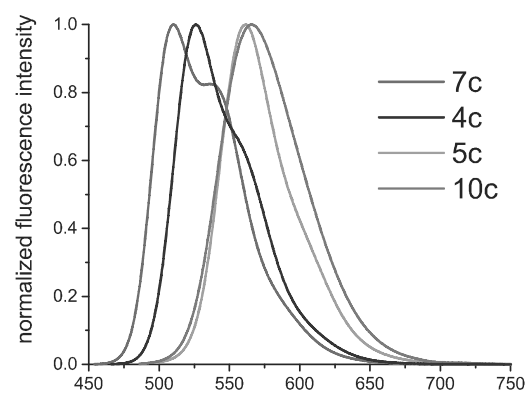

wavelength $\lambda / \mathrm{nm}$

Figure 1. Chemical structures of the open and the closed form of the photoswitches 1oc-10oc; fluorescence spectra of 4c, 5c, 7c, and 10c in 1,4-dioxane (for spectra of other derivatives see the Supporting Information).

efficiently. It is, however, not only the good fluorescence quantum yield that accounts for the power of the dyes for super-resolution imaging, but their switching behavior which will be discussed as follows.

\subsection{Photoswitching}

The open-ring isomer and the closed-ring isomer can be converted into each other by irradiation with suitable wavelengths

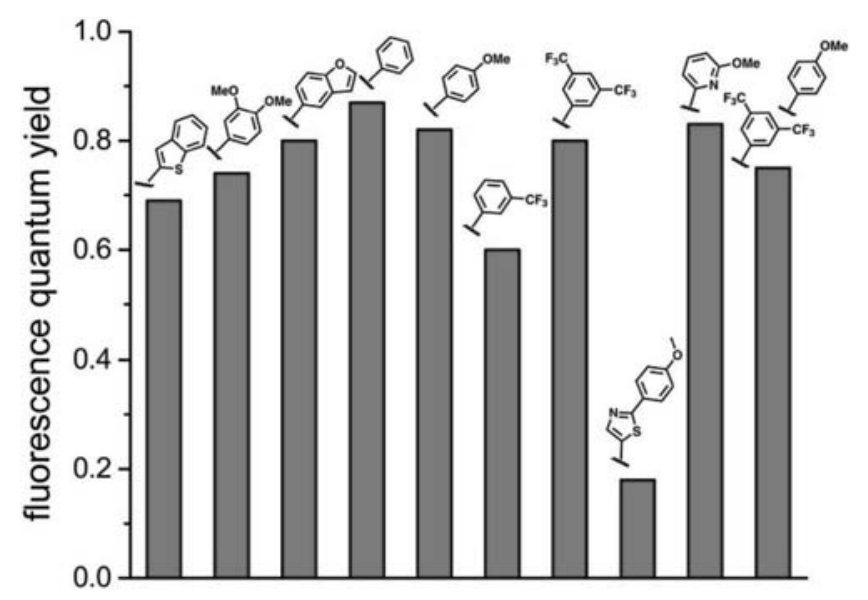

Figure 2. Histogram of fluorescence quantum yields of compounds $1 \mathrm{c}-10 \mathrm{c}$ in 1,4-dioxane. (see Figure 3). Irradiation with UV light results in the formation of the closed form, while the visible light irradiation with wavelengths smaller than $570 \mathrm{~nm}$ leads to the recovery of the open-ring isomer. After sufficiently long irradiation, the photostationary state is reached which depends on the absorption coefficient of both forms at the corresponding irradiation wavelength and their cycloconversion/cycloreversion quantum yields.

For a good localization accuracy in localization-based superresolution and image-correlation methods, a fluorophore has to emit several thousands of photons before off-switching. ${ }^{[4]}$ Therefore, one key property for photoswitches in this application is a sufficiently low off-switching quantum yield. Several diarylethene derivatives presented here fulfill this requirement with cycloreversion quantum yields below $\approx 10^{-4}$. In contrast, for RESOLFT microscopy, the photoswitching quantum yield is of minor importance. Here, it is more important to prevent photobleaching and to allow for switching over many cycles. The on-switching quantum yields of the photoswitches presented here are reasonable high so that they do not set practical limits for any of the super-resolution methods investigated here. In the following, compound $50 \mathrm{c}$ will be discussed in detail (corresponding figures and data for all other switches can be found in the Supporting Information). Its quantum yield $\phi_{0 \rightarrow c}$ for the cyclization reaction was determined to be 0.13 in 1,4-dioxane (see Figure 3 and Table S3, Supporting Information). Thus, the ring-closing photoreaction to switch on its fluorescence is very photon-efficient. The off-switching, since it is rather inefficient, had to be determined with a high power $488 \mathrm{~nm}$ laser and yielded a quantum yield $\phi_{c \rightarrow 0}$ of $3.2 \times 10^{-6}$, i.e., statistically 

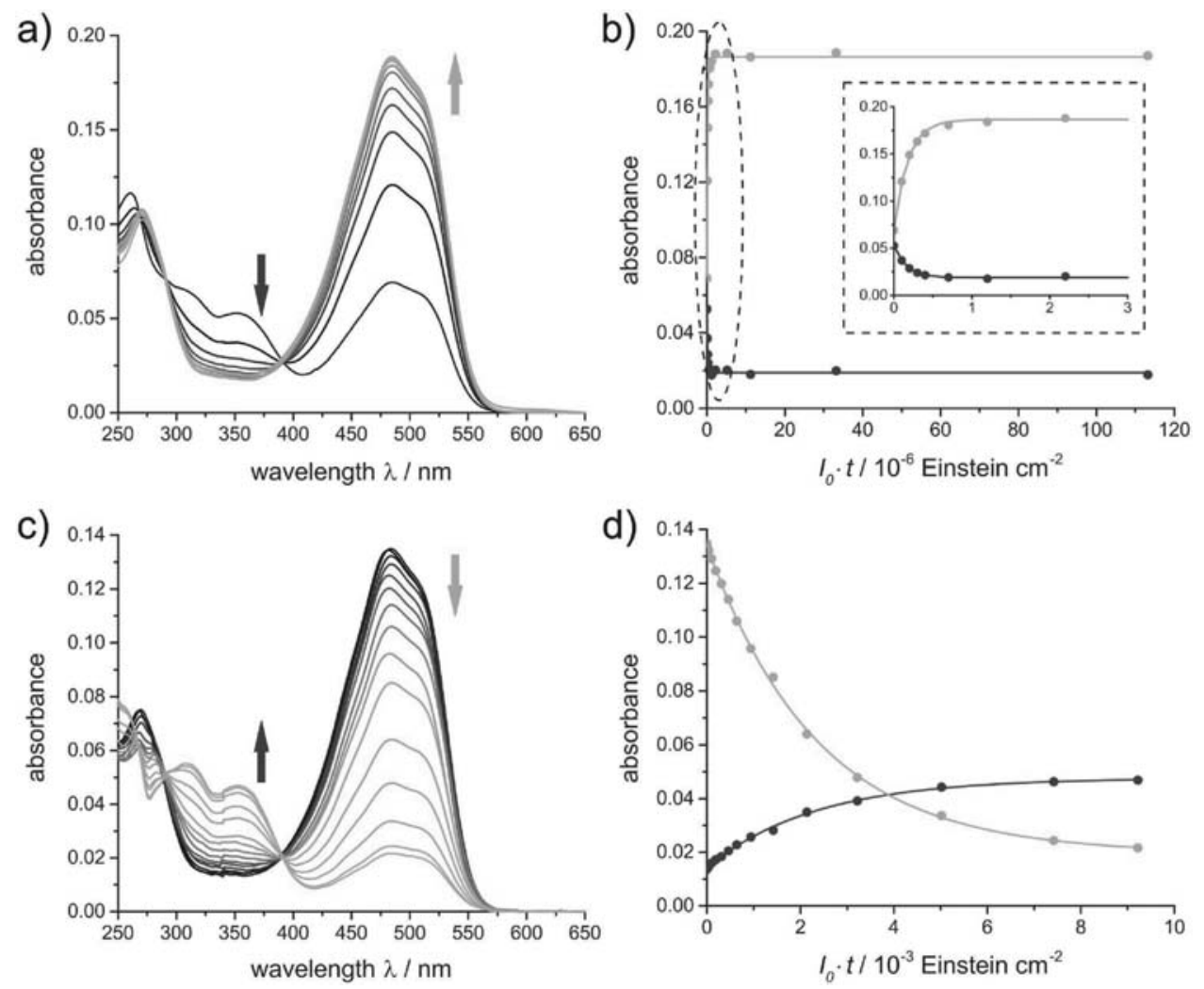

Figure 3. a) Switching from the open 50 to the closed $5 \mathrm{c}$ form under UV light irradiation ( $366 \mathrm{~nm}$ ) until reaching the photostationary state (almost exclusively the closed form) as determined by UV-vis absorption and NMR spectroscopy; b) time-evolution of the absorbance at $485 \mathrm{~nm}$ (green circles) and at $353 \mathrm{~nm}$ (blue circles) upon irradiation with $366 \mathrm{~nm}\left(I_{0}=10^{-7}\right.$ Einstein $\left.\mathrm{cm}^{-2}\right)$, the inset shows a zoom area of the first $\left.3 \mathrm{~s} ; \mathrm{c}\right) \mathrm{switching}$ from the closed $5 \mathrm{c}$ to the open 50 form under irradiation with $494 \mathrm{~nm}$ until reaching the photostationary state; d) time-evolution of the absorbance at $485 \mathrm{~nm}$ (green circles) and at $353 \mathrm{~nm}$ (blue circles) upon irradiation with $488 \mathrm{~nm}\left(I_{0}=10^{-6}\right.$ Einstein $\left.\mathrm{cm}^{-2}\right)$.

the off-switching proceeds only after $\approx 300000$ excitations. The extremely low quantum yield for the off-switching is one of the most essential advantages of the presented DAE for PALM imaging since it ascertains that enough photons are collected for a high accuracy in the localization of the positions of single photoswitches before converting to the off-state.

Switching and fluorescence properties of the photoswitches 1oc-10oc in 1,4-dioxane are summarized in Table S3 of the Supporting information. By attaching different types of substituents, the positions of the absorption and fluorescence bands of both open-ring and closed-ring isomers, as well as cycloconversion and cycloreversion quantum yields can be tuned easily. It was recently shown that introducing electron withdrawing substituents to diarylethene photoswitches increases their cycloreversion quantum yield. ${ }^{[45,46]}$ The opposite effect is obtained when electron donating groups are present, mainly due to a better stabilization of the delocalized $\pi$-system with respective substituents. ${ }^{[47]}$ For compounds $10 \mathrm{c}$, 2oc, and 5oc, the absorption bands of both the open-ring and closed-ring isomer, are red-shifted compared to those of the phenyl derivative 4oc. In addition, the cycloreversion quantum yields drop by a factor of more than 400. A determination of the cycloreversion quantum yield was even not possible for compounds $10 \mathrm{c}$ and $2 \mathrm{oc}$ due to a very slow ringopening reaction. Introducing electron withdrawing groups, such as trifluoromethyl, increases the cycloreversion quantum yields. Compound 7oc switches ten times faster to the open form than compound 4oc. Typical "push-pull" chromophores contain in their structure both an electron withdrawing and an electron donating moiety separated from each other by a $\pi$-system. As a result, in the excited state a charge transfer from the electron rich part to the electron poor part could be observed. ${ }^{[48]}$ In case of the diarylethene photoswitches under investigation this approach has two main advantages. First, it shifts the absorption bands of the closed form bathochromically, thus enabling their excitation with longer wavelengths. Second, it increases the cycloreversion quantum yield in comparison to the corresponding symmetric photoswitch with the same electron donating moieties. These properties can be demonstrated with compound 10oc. Compared to 5oc, its closed-ring absorption maximum is shifted by $9 \mathrm{~nm}$ and the cycloreversion quantum yield is increased by one order of magnitude from $3.2 \times 10^{-6}$ to $4.1 \times 10^{-5}$. Thus, choosing the right substituents allows for tuning the diarylethene photoswitch and optimizing it for different super-resolution techniques. Low cycloreversion quantum yields are favored for PALM, whereas the possibility to switch several times with a low fatigue is of advantage for RESOLFT.

\subsection{Dependency of Photoswitching on Polymer Type}

The choice of a suitable photoswitch derivative does not only depend on the technique to be used but also on the structure 


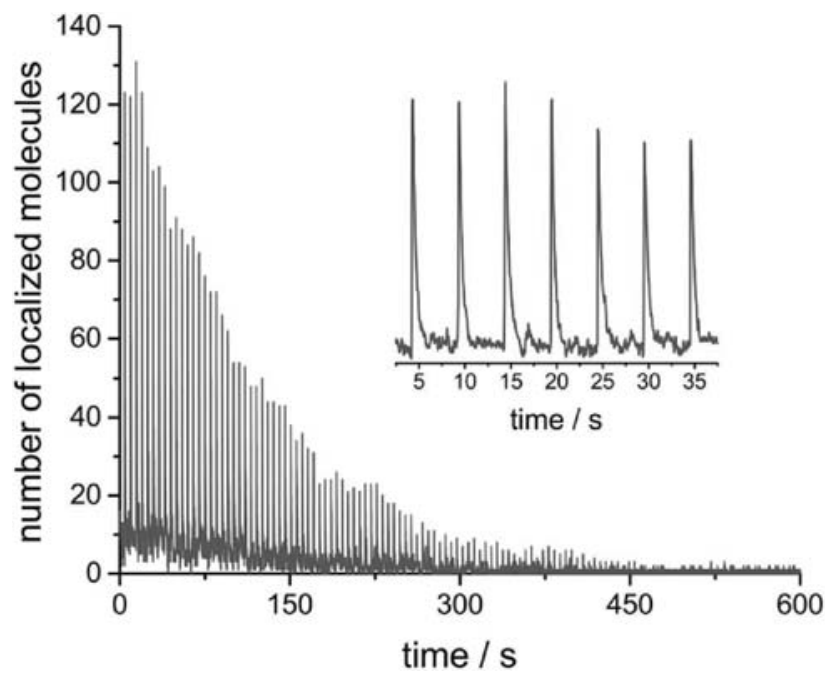

Figure 4. Switching cycles of compound $50 \mathrm{oc}$ embedded in a thin PEA film upon $488 \mathrm{~nm}\left(0.5 \mathrm{~kW} \mathrm{~cm}^{-2}\right)$ excitation and UV-activation every $5 \mathrm{~s}$. The inset shows a zoom into the first $30 \mathrm{~s}$ interval, where individual offswitching cycles can be recognized. In contrast to PALM imaging, the UV activation laser power was chosen sufficiently high to activate basically all (nondamaged) photoswitches at the beginning of each cycle.

to be labeled. Especially when labeling dense polymer phases, the photoswitching kinetics can be significantly influenced. One important parameter describing the polymer density and thus the free volume available for the photoswitch for its cyclization or cycloreversion is its glass transition temperature $T_{\mathrm{g}}$ as has been demonstrated, e.g., by studies on photoinduced electron transfer. ${ }^{[49,50]}$ Irie and co-workers describe the dependency of the DAE switching kinetics on the environment, ${ }^{[51]}$ and we previously observed a reduced switching behavior in highly viscous media as well.[26] We investigated the switching behavior of the photoswitches embedded in poly(ethylacrylate) (PEA), poly(n-butylmethacrylate) (PBMA), poly(styrene) (PS), and poly(methylmethacrylate) (PMMA) thin polymer films, which were prepared by spin-coating (for the experimental procedure see the Supporting Information). As an example, the switching behavior of compound 5oc in a PEA film is shown in Figure 4. We have counted the number of photoswitches in the fluorescent on-state by analyzing a $40 \times 40 \mu^{2}$ area of a widefield microscopy image which was continuously excited with a $488 \mathrm{~nm}$ laser $\left(0.5 \mathrm{~kW} \mathrm{~cm}^{-2}\right)$ while the photoswitches were activated with a $380 \mathrm{~nm}$ UV laser pulse (duration $0.1 \mathrm{~s}$, intensity $\approx 0.6 \mathrm{~kW} \mathrm{~cm}^{-2}$ ) every $5 \mathrm{~s}$. For all four polymers more than 100 switching cycles could be observed (see Figures S12-S14 of the Supporting Information). Due to photobleaching the total number of localized molecules in each individual cycle decreases (see Figure 4 inset). A detailed analysis shows distinct switching kinetics of diarylethene photoswitches in the different polymers. The individual switching cycles of 50 in PEA, PBMA, PMMA, and PS are shown in Figure 5a. For each measurement series, the first ten cycles were taken for analysis and normalized by the following procedure. Normalization was performed by dividing the number of the localized molecules $N_{\text {on }}$ at a certain time by the number of localized molecules $N_{\mathrm{on}, 0}$ at the beginning of the respective cycle. The temporal evolution of the number of photoswitches in their on-state strongly depends on the polymer. In

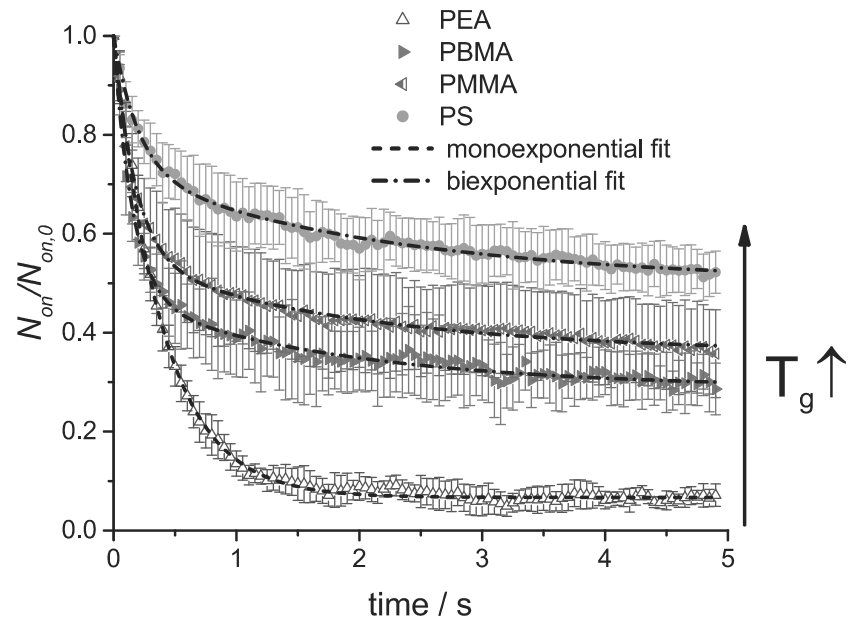

Figure 5. Temporal evolution of the number of photoswitches in the onstate at room temperature for compound $50 \mathrm{c}$ in PEA, PBMA, PMMA, and PS polymer films, respectively, upon $488 \mathrm{~nm}\left(0.5 \mathrm{~kW} \mathrm{~cm}^{-2}\right)$ excitation and UV-activation every $5 \mathrm{~s}$ for PEA, PBMA, and $10 \mathrm{~s}$ for PMMA, PS for $0.1 \mathrm{~s}$ $\left(0.6 \mathrm{~kW} \mathrm{~cm}{ }^{-2}\right)$, each. The reason for the offset is explained in the text.

films of the low $T_{\mathrm{g}}$-polymer PEA, first order kinetics with its typical monoexponential behavior was observed and the corresponding rate constant for off-switching was determined. For the other polymers, the cycloreversion can be described with biexponential kinetics (see Figure 5). Higher off-switching rates in polymers of low $T_{\mathrm{g}}$ were observed because of less freedom for rotating around chemical bonds at $T=T_{\mathrm{g}}$. DAE undergo the cyclization reaction only when they are in the antiparallel confirmation. ${ }^{[36]}$ This might be an explanation why in higher $T_{\mathrm{g}}$ polymers a fraction of molecules does not switch at all and an offset in the fits in Figure 5 occurs. From single molecule FM measurements, ${ }^{[52]}$ it is known that the free volume can be distributed heterogeneously. Thus, dyes in the vicinity of more free volume might be able to reach the antiparallel conformation while others are fixed rigidly in the polymer. A way to overcome this problem in the context of SRI is discussed below.

The dependency of the switching kinetics on the proximity to the glass transition temperature can either be shown by measurements in different polymers, or can be rationalized with measurement in one polymer at different temperatures. We chose PBMA to quantify this behavior and present the results in Figure S15 in the Supporting Information. Indium tin oxide (ITO) ${ }^{[53]}$ coverslips with photoswitch 5 oc embedded in a thin PBMA film on their top were prepared by spin-coating as described in the Supporting information. These samples were measured from room temperature up to $T_{\mathrm{g}}+20^{\circ} \mathrm{C}$, and the photoswitching behavior at each temperature was determined (see Figure S16 in the Supporting Information). Increasing the temperature shows the transition from biexponential switching kinetics to a monoexponential first order kinetics. The offswitching (cycloreversion) rate constants and quantum yields at different temperatures were calculated and are presented in Table S3 in the Supporting Information. The off-switching quantum yield is almost two times higher at $60{ }^{\circ} \mathrm{C}$ than at room temperature.

As it was shown before, ${ }^{[54-56]}$ single molecules in polymer matrices can rotate at temperatures higher than $T_{\mathrm{g}}$. This 
corresponds to our observations of DAE switching kinetics. At high temperatures, molecules have more freedom to rotate which results in higher cycloreversion quantum yields. In rigid polymer matrices (PS and PMMA), the differences in off-switching quantum yields of compounds 4oc, 5oc, 7oc, and $10 \mathrm{oc}$, representing different substitution types, are less pronounced compared to measurements in solution (Figure S17, Supporting Information). Compound $50 \mathrm{c}$ with electron donating $\mathrm{MeO}$-groups switches back to the open form only six times slower than compound 7oc with strong electron withdrawing $\mathrm{CF}_{3}$-groups, whereas it was a factor of 1000 in solution. We conclude that for localization-based super-resolution microscopy methods, investigating rigid polymer systems, DAEs irrespective of the substituent type can be used. But at the same time, the switching kinetics becomes more crucial for softer materials, and for deterministic methods such as RESOLFT fast switching dyes are preferable.

\subsection{Photon Budget}

The number of photons detected from fluorophores is one of the essential parameters to be optimized for good superresolution microscopy. We determined the photon budget of the photoswitchable DAE in thin PS and PEA films as described in the Supporting Information. The number of photons directly depends on the switching kinetics of the photoswitch in polymer matrices, which was described in the previous section. For the derivatives $50 \mathrm{c}$ and $10 \mathrm{oc}$ with electron donating substitutes, we obtained on average 775 and 1020 photons per on-cycle in PS, respectively. In contrast, the derivatives $60 c$ and $70 c$ with electron withdrawing groups only yielded $\approx 500$ photons because of more efficient switching to the offstate (for the detailed information see Figure S18 in the Supporting Information). The photon number per cycle depends also on the polymer environment. In PEA which is softer than PS (lower $T_{\mathrm{g}}$ ), the photon budget becomes approximately two times larger for all investigated photoswitches (see Figure S19 in the Supporting Information). As a consequence of this complex behavior of these photoswitches in polymer matrices, numerous different effects such as switching kinetics and local polarity of the polymer have to be taken into account for choosing the optimal derivative for the corresponding method. Thus, for example, for the investigation of soft polymer structures it is advantageous to combine either stochastic super-resolution methods with photoswitches bearing electron donating substituents, or deterministic methods with photoswitches bearing electron withdrawing groups. In contrast, for rigid structures, the choice of the fluorophore is less important due to the similar switching behavior of the different photoswitches.

Another important aspect concerning the suitability of a fluorescent probe for super resolution microscopy is its photostability. As shown above, DAE can survive several hundreds of on-off switching cycles (see Figure 4) before photobleaching due to irreversible photodamage of the molecule. This is an advantage in comparison with other small organic photoswitchable dyes, especially when aiming for an investigation of structural changes over time.

\subsection{Super-Resolution Imaging}

\subsubsection{PALM}

Diarylethene photoswitches are suitable for PALM imaging, as we have demonstrated in our previous work where we labeled apolar structures. ${ }^{[26]}$ Analogously, Hell and coauthors applied water-soluble diarylethene for biological studies, i.e., visualizing labeled microtubules. ${ }^{[40]}$

For our study described here, we used two different types of block copolymers with a hydrophobic and a hydrophilic block. Both polymers form cylindrical micelles and contain a polyethylene oxide (PEO) block. Details of the labeling procedure are presented in the Experimental Section. As the apolar block, two polymers with rather different glass transition temperatures, i.e., PS with a bulk- $T_{\mathrm{g}}$ of $100{ }^{\circ} \mathrm{C}$ and poly$\varepsilon$-caprolactone (PCL) with a bulk- $T_{g}$ of $-69^{\circ} \mathrm{C}$, were chosen. Under appropriate conditions, self-assembled structures of amphiphilic polystyrene-polyethylene oxide (PS- $b$-PEO, $M_{\mathrm{n}, \mathrm{PS}}=$

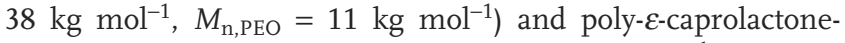
polyethylene oxide (PCL- $b$-PEO, $M_{\mathrm{n}, \mathrm{PCL}}=10 \mathrm{~kg} \mathrm{~mol}^{-1}, M_{\mathrm{n}, \mathrm{PEO}}=$ $5 \mathrm{~kg} \mathrm{~mol}^{-1}$ ) block copolymers with a hydrophobic core consisting of PS or PCL, respectively, and a hydrophilic PEO shell could be formed. The core was labelled with noncovalently embedded fluorescent diarylethene derivatives. A drop of solution was spin-coated and dried, yielding cylindrical block copolymer structures on a coverslip. FM movies were recorded under appropriate laser intensities to allow for a well-defined single photoswitch blinking kinetics (for details see Supporting Information). As shown before (see Figure 5), some dyes do not switch efficiently within very rigid polymers such as PS with high $T_{\mathrm{g}}$. Therefore, these photoswitches were photobleached with the $488 \mathrm{~nm}$ laser before the actual PALM measurements. Analysis with ThunderSTORM ${ }^{[57]}$ generated super-resolved PALM images are shown in Figure 6a and Figure S21 in the Supporting Information. In the super-resolved part of the images, structural details can be resolved which are obscured in the diffraction-limited part. No significant difference between switching properties of different diarylethene derivatives in rigid polymers was detected (see the comparison between 6oc (Figure 6) and 10oc (Figure S21 in the Supporting Information) labeled PS-b-PEO. For the sample labeled with $10 \mathrm{oc}$, we additionally performed Fourier ring correlation (FRC) ${ }^{[58]}$ analysis to determine a spatial resolution of $45 \mathrm{~nm}$ (see Figure S22 in the Supporting Information). We also ascertained a sufficiently high labeling density to fulfil the Nyquist criterion. ${ }^{[59]}$ For example, two micelles which are $70 \mathrm{~nm}$ apart from each other can be easily resolved (see Figure S21b in the Supporting Information). Recently, Miyasaka and co-workers showed a one color control of the cycloreversion/cycloconversion process of a fluorescent diarylethene due to the excitation of a "hot band" of the open-ring isomer. ${ }^{[41]}$ We decided to apply the same concept for PALM imaging of polymer nanostructures. The possibility of using only one laser for super-resolution imaging and switching control at low laser power conditions opens great opportunities for using super-resolution microscopy in polymer science using a rather simple microscopy setup. The image of cylindrical micelles labeled with 6oc obtained with irradiation at $488 \mathrm{~nm}$ only, i.e., without UV activation, in Figure $6 \mathrm{~b}$ has a 


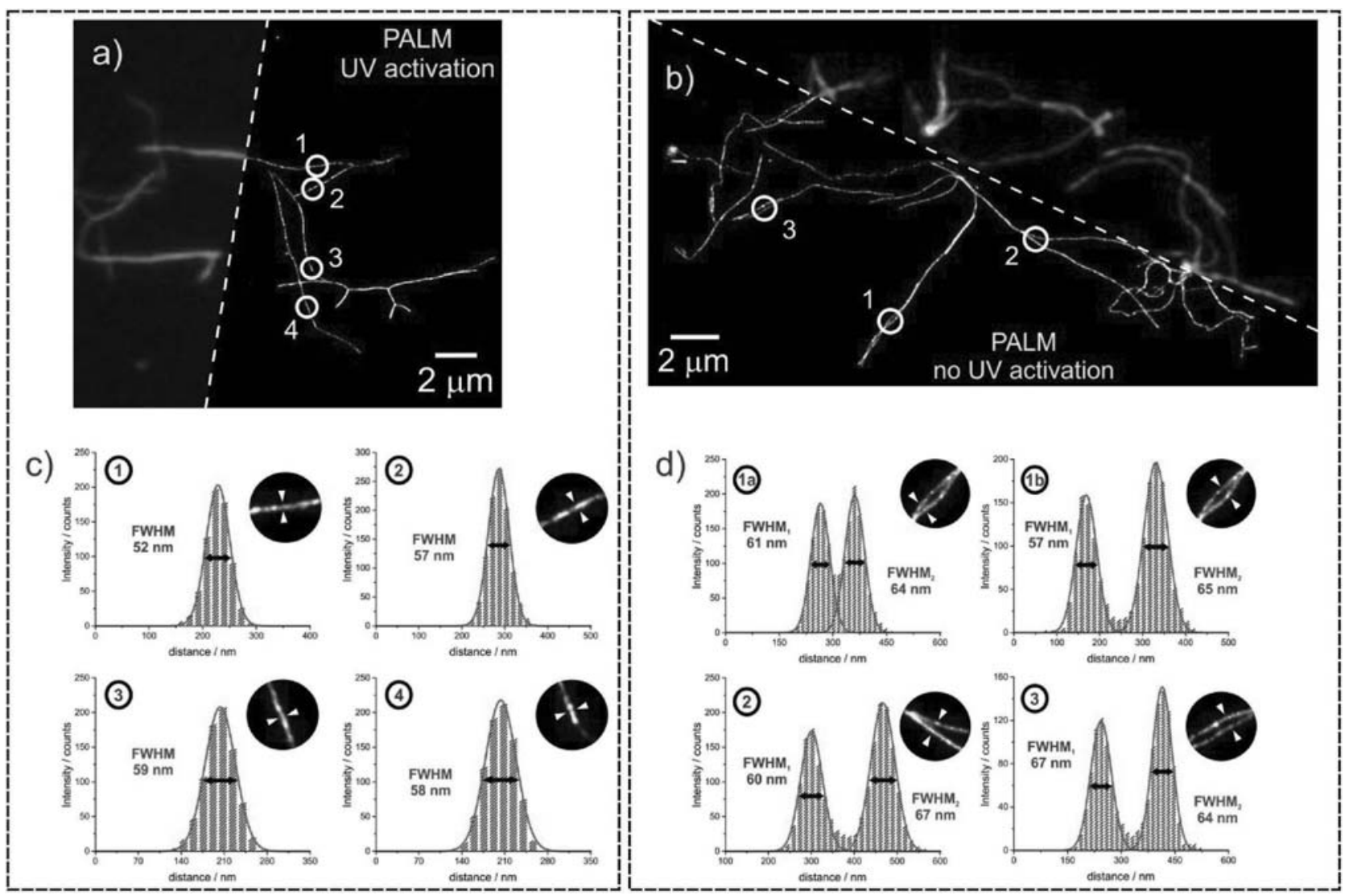

Figure 6. a) Conventional (left part) wide-field and super-resolved PALM (right part) image of PS- $b$-PEO block copolymer self-assembly structures labeled with 6oc; excitation was performed with $488 \mathrm{~nm}$ laser $\left(6.25 \mathrm{~kW} \mathrm{~cm}^{-2}\right)$ and UV-activation was done with $380 \mathrm{~nm}$ laser (0.6 kW $\left.\mathrm{cm}{ }^{-2}\right)$; b) The same experiment as in (a) except that no UV excitation was applied. c,d) Zoom of different areas in the PALM super-resolved structures with intensity profiles across the individual cylindrical micelles are presented.

similar quality as the image with UV activation in Figure 6a. Figures $6 c$,d demonstrates the capability to resolve structures well below the diffraction limit in both cases. The average size of the cylinders as extracted from the image is around $60 \mathrm{~nm}$ with respect to full-width at half-maximum. Since the cylinders, however, possess a non-negligible width of $\approx 50 \mathrm{~nm}$, and the observed sizes are a convolution of the real size of the labeled core with the single molecule localization accuracy, we can estimate our resolution to be $\approx 47 \mathrm{~nm}$.

\subsubsection{SOFI}

Despite its good resolution, the drawback of PALM is the time it requires to obtain sufficient single molecule localizations to resolve a structure. Thus, only structural changes in the multiseconds to minutes range can be reasonably well followed, although their observation would be highly interesting in polymer science. ${ }^{[60]}$ The principal possibility to follow in situ dynamics is, however, one of the main advantages of (superresolution) FM compared to AFM and EM methods, and thus can open new horizons. SOFI offers a powerful alternative for faster imaging, providing, however, a lower spatial resolution than that of PALM. The main requirements for SOFI are high labeling density and well adjustable switching kinetics. While a low off-switching quantum yield is essential for PALM, this condition is less important for SOFI. We compared PALM and SOFI of the same nanostructure labeled with 6oc (see Figure 7). The resolution of the PALM image in Figure $7 \mathrm{a}$ is $63 \mathrm{~nm}$ as calculated with FRC (see Figure S23 in the Supporting Information). The second order SOFI analysis was performed using a custom-made analysis-routine. ${ }^{[61]}$ We also provide a comparative analysis of different numbers of frames in Figure 7c. Results close to the theoretical optimum of SOFI were obtained for 5000 frames using an improved analysis algorithm (see Figure 7b; for a description of the analysis see the Supporting Information). The resolution calculated for this image taking into account the point spread function of the single emitters is about $115 \mathrm{~nm}$. Compared to PALM, the resolution is decreased by a factor of 2 , but acquiring this image was two orders of magnitude faster. A reasonably well resolved SOFI image (Figure $7 \mathrm{~b})$ was recorded within $2 \mathrm{~s}(200$ frames, $10 \mathrm{~ms}$ integration time), whereas the PALM image of Figure 7a required more than $4 \mathrm{~min}$ (25 000 frames, $10 \mathrm{~ms}$ integration time) to be recorded. A further example of SOFI of labeled self-assembly nanostructures, with a comparison between second and third order analyses, can be found in the Supporting Information (see Figure S26 in the Supporting Information). Compared to second order SOFI with a maximum resolution gain of a factor of two, the third order SOFI 

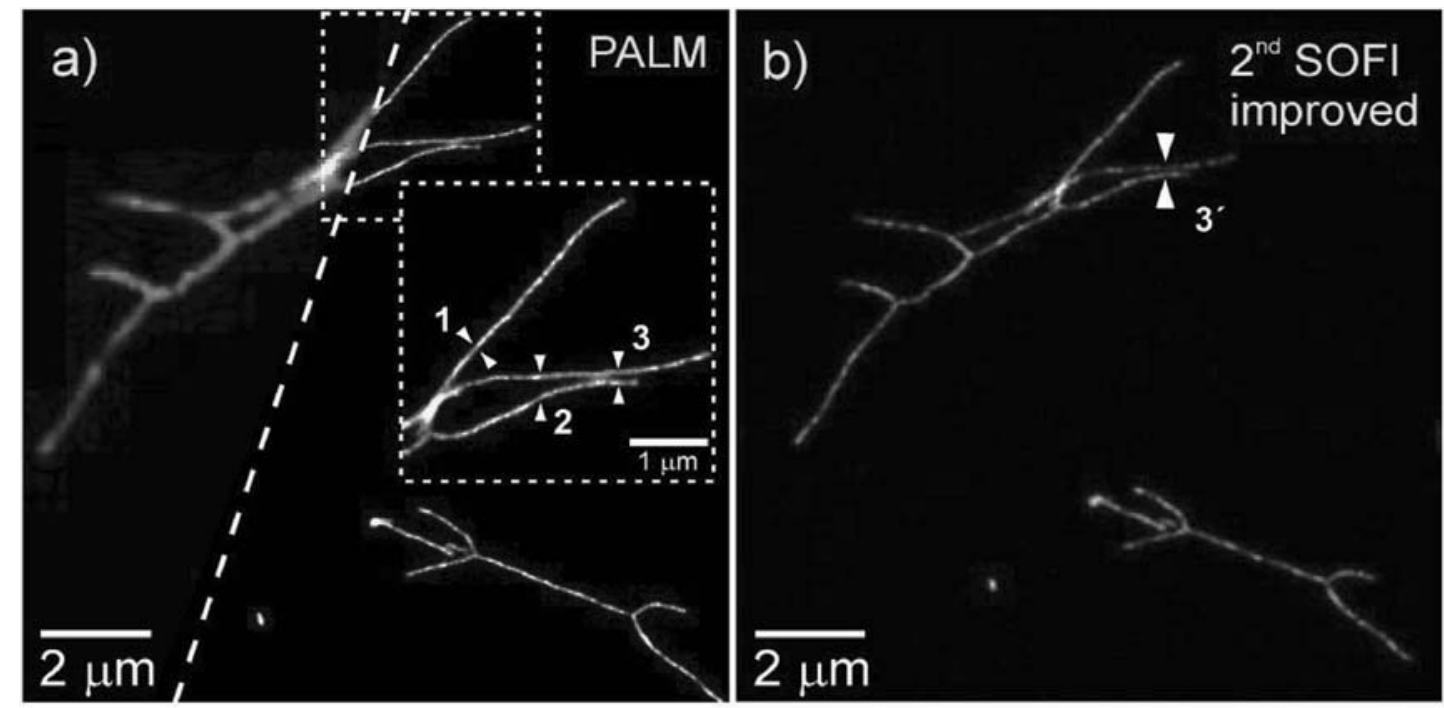

c)
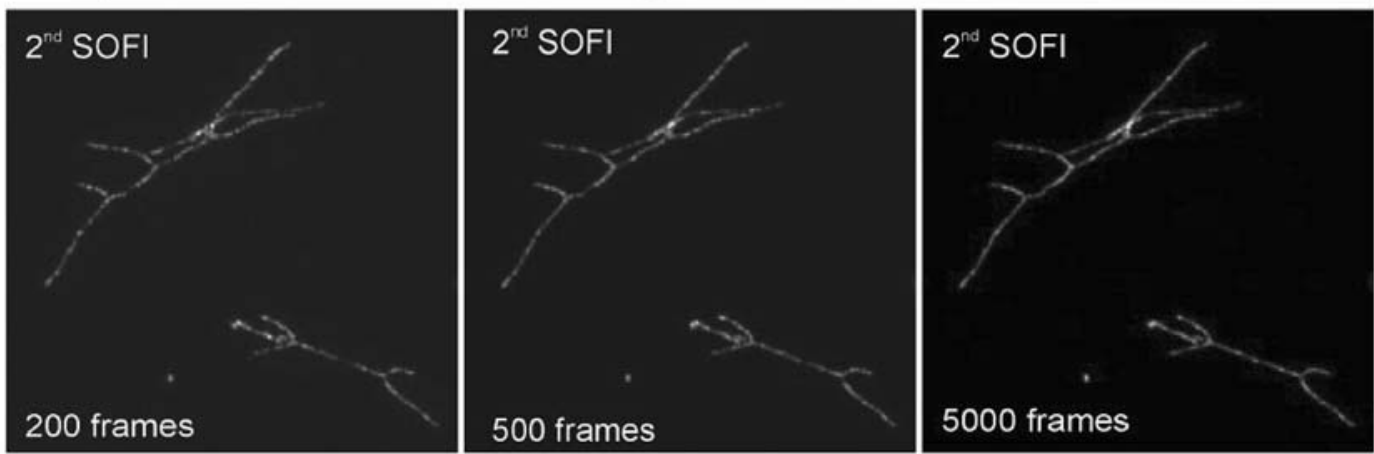

d)
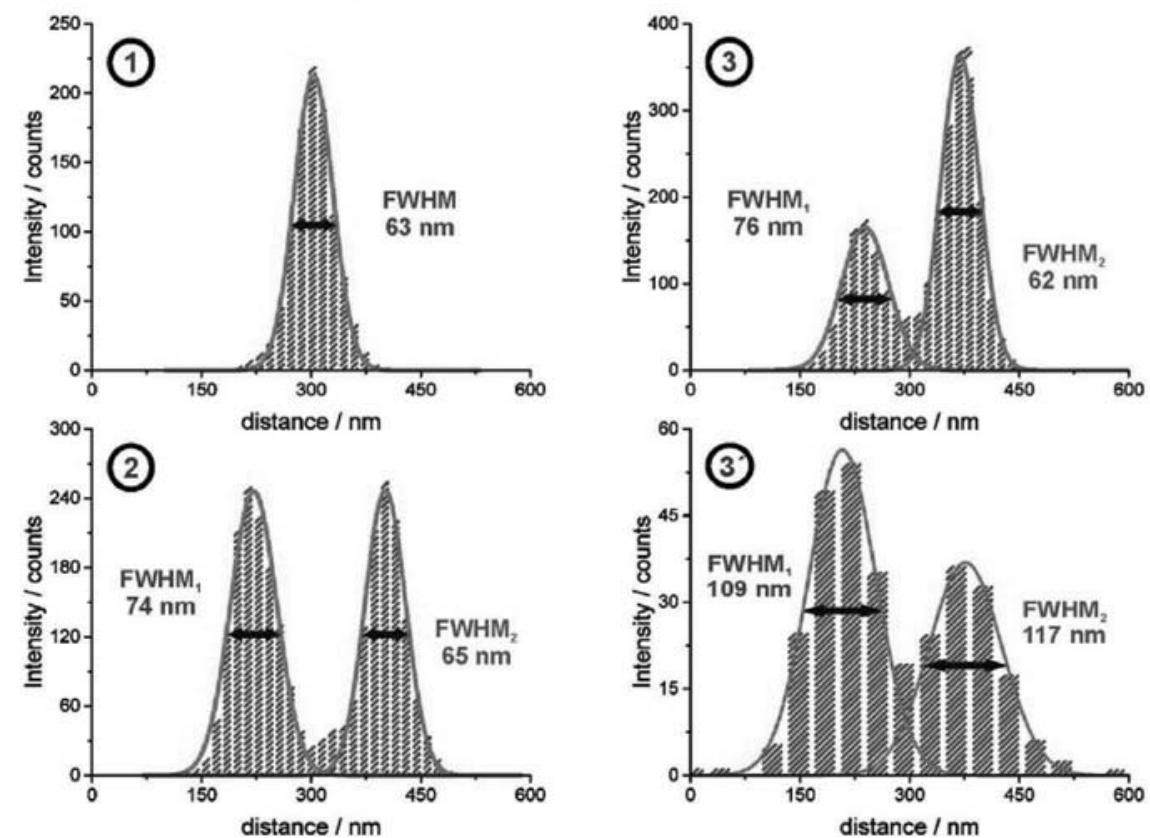

Figure 7. a) Conventional wide-field (left part, 25000 frames analyzed) and super-resolved PALM (right part, 5000 frames analyzed) image of the PS- $b$-PEO block copolymer self-assembly structures labeled with 6oc, the inset shows a zoomed area; excitation was performed with a 488 nm laser $\left(0.2 \mathrm{~kW} \mathrm{~cm}{ }^{-2}\right)$ without UV-activation; b) second order SOFI super-resolved improved image of the same region of interest; excitation was performed with $488 \mathrm{~nm}$ laser $\left(0.2 \mathrm{~kW} \mathrm{~cm}{ }^{-2}\right)$ and $405 \mathrm{~nm}$ UV-activation $\left(2 \mathrm{~W} \mathrm{~cm}^{-2}\right)$ was used; c) second order SOFI super-resolved images as a result of the analysis of 200, 500, 5000 frames; d) intensity profiles of the individual cylindrical micelles of the corresponding PALM image in (a). In addition, a comparison between the resolution of two cylindrical micelles in the SOFI image (labeled with 3') with 200 frames in (c) and the corresponding two cylindrical micelles in the PALM image (labeled with 3) in (a) is drawn. 
shows a 2.5 -fold improvement in spatial resolution, but is also more prone to artifacts.

\subsubsection{RESOLFT}

RESOLFT imaging, unlike PALM and SOFI, is based on the deterministic switching between long-living molecular states, i.e., a fluorescent on- and a nonfluorescent off-state. Thus, the ideal fluorophore should not only be bright when in its on-state, but it should also feature a high number of on-off cycles, a requirement that is fulfilled for the diarylethene photoswitches presented here. Reversibly switchable fluorescent proteins are currently the standard for RESOLFT imaging due to the low switching fatigue and optimized on-off kinetics. ${ }^{[20,21,62]}$ However, advances in RESOLFT imaging and its application in polymer science will benefit from bright organic fluorophores.

Here, we demonstrate the potential of the diarylethenes compounds for RESOLFT imaging (Figure 8) with the same dye concentrations as used for PALM and SOFI. RESOLFT images were recorded by (1.) switching on a dye population with a $405 \mathrm{~nm}$ Gaussian shaped beam and (2.) by sequentially
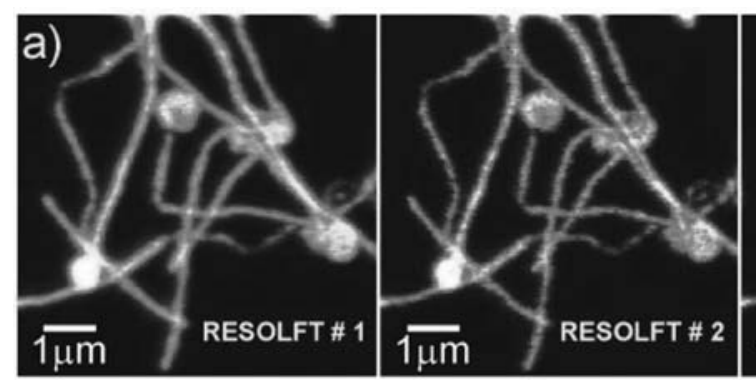

b)

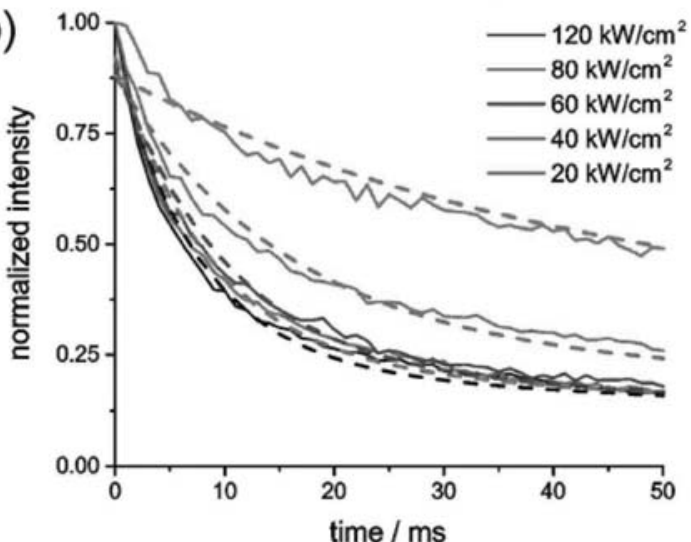

d)

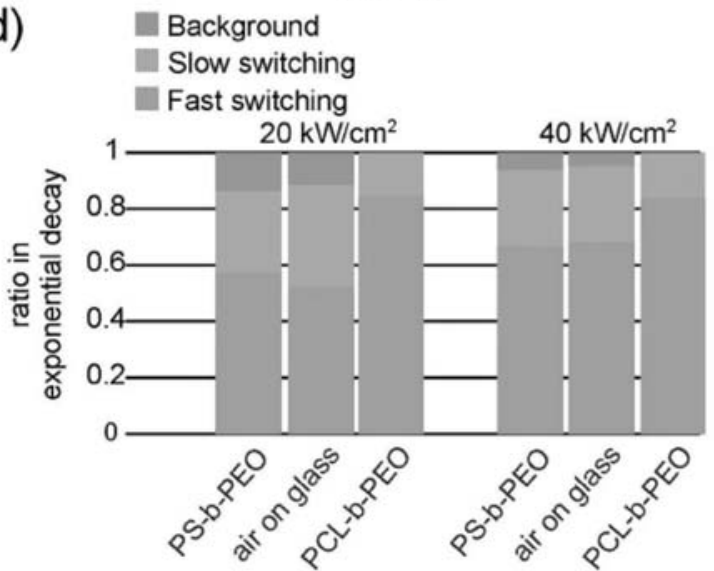

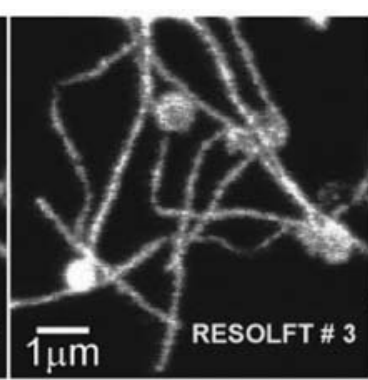

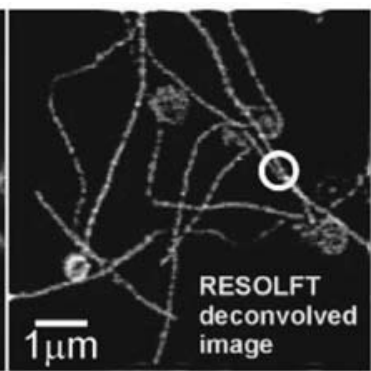

c)

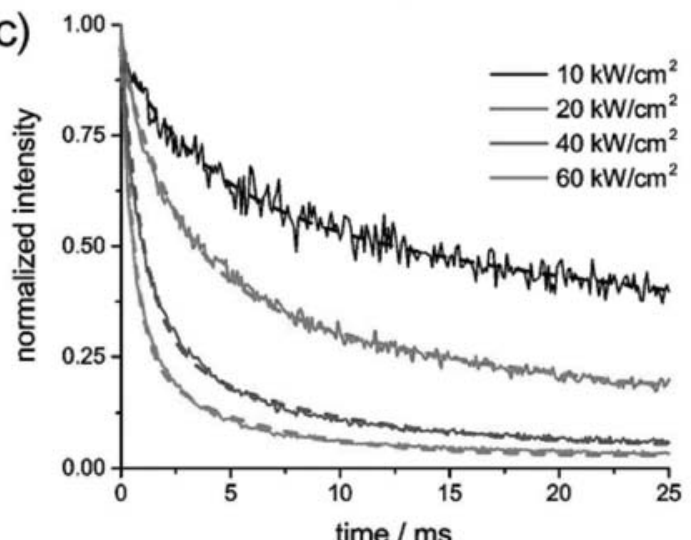

e)

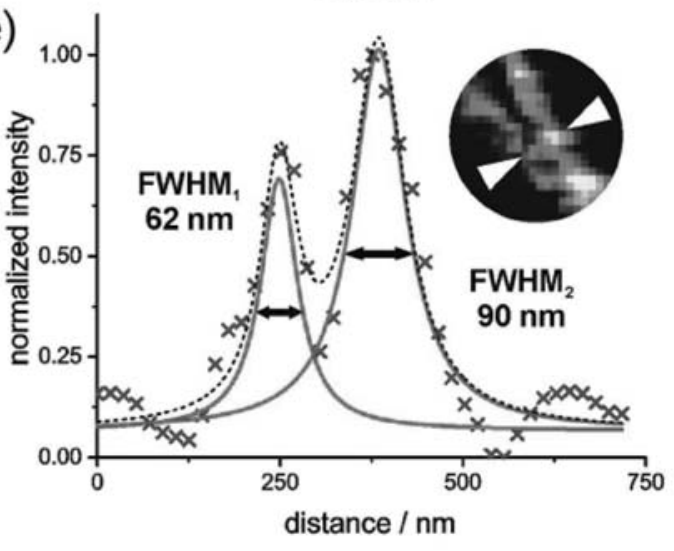

Figure 8. RESOLFT imaging and on-off switching kinetics. a) Sequentially recorded RESOLFT images of PS- $b$-PEO block copolymer self-assembly structures labeled with 6oc; the images where recorded with on-switching of $24 \mathrm{~kW} \mathrm{~cm}{ }^{-2}$ of a $405 \mathrm{~nm}$ laser for $0.2 \mathrm{~ms}$, followed by $40 \mathrm{~kW} \mathrm{~cm}$-2 of a donut-shaped $488 \mathrm{~nm}$ laser $5 \mathrm{~ms}$, and finally $0.2 \mathrm{~ms}_{20 \mathrm{~kW} \mathrm{~cm}}^{-2}$ of a $488 \mathrm{~nm}$ laser for the read-out phase; b) the off-switching kinetics of $60 \mathrm{c}$ in PS$b$-PEO micelles at different intensities of $488 \mathrm{~nm}$ laser light. The dashed lines represent a two components exponential decay fit; c) the off-switching kinetics of $60 \mathrm{c}$ on the glass surface at different $488 \mathrm{~nm}$ laser intensities; the dashed lines represent a 2 components exponential decay fit; d) the corresponding decays represent three different stages—a fast switching, a slow switching and a background; e) zoom into the RESOLFT super-resolved structures with line profiles across the individual cylindrical micelles. 
switching the population of dyes located in the periphery of the focal spot into a dark state with a donut shaped beam. Finally, (3.) the dyes left in the on state are excited and recorded with a Gaussian shaped beam at $488 \mathrm{~nm}$. This scheme of pulses is applied pixel by pixel to build up a super resolved image of the dye in a PS- $b$-PEO block copolymer.

For an optimal selection of imaging parameters for the RESOLFT pulse scheme, it is essential to investigate the switching behavior of the dyes. The kinetics was measured in a pump-probe mode by shortly illuminating the dyes using a $405 \mathrm{~nm}$ laser to drive them to the on-state, followed by extended exposure with $488 \mathrm{~nm}$ light to measure the off-switching kinetics. A selection of results of these experiments for different intensities of the $488 \mathrm{~nm}$ laser illumination is shown in Figure 8b,c. The off-switching kinetics is bi-exponential and reveals a background fluorescence. Both time constants are inversely proportional to the off-switching intensities. The fast half-life $\left(t_{t / 2}\right)$ is $t_{1 / 2}<10 \mathrm{~ms}$ for all but the lowest power, and the slow switching constant is $t_{1 / 2}>80 \mathrm{~ms}$. The ratio between the fast, slow, and nonswitching (background fluorescence) components of the dye is shown in Figure 8d. For the PS- $b$-PEO block copolymer, the fast switching component constitutes about $70 \%$ across all powers examined. This knowledge can be used to extract more spatial information in the RESOLFT images. In fact, a closer comparison between the three sequential images reveals a notable increase in resolution at the later images, with a large difference between the first and second image. This is likely caused by photobleaching of the nonswitching component, i.e., the background. The last image of Figure 8a is deconvolved with a PSF accounting for the background fluorescence. Looking at the final deconvolved image, sub-resolved features can easily be observed, such as structures with widths of about $60 \mathrm{~nm}$ (line profile in Figure 8e).

The switching kinetics proved to be highly dependent on the local environment, as can also be seen by observing the differences in Figure 8b,c. In Figure 8c the "stiff" polymer has been exchanged with a free air environment, with the dye simply being spin-coated onto a glass substrate. The free dye shows an off-switching kinetics that is about 20 times faster than that for dyes in the PS- $b$-PEO polymer, for both the fast and slow switching stage. Interestingly, the ratio between the three components seems to be mostly unaffected, as can be seen in Figure $8 \mathrm{~d}$. However, when the dyes are embedded into a softer poly- $\varepsilon$-caprolactone core of a PCL- $b$-PEO structure, the offswitching kinetics is also $\approx 20$ times faster (see the Supporting Information). Additionally, the background fluorescence has almost completely vanished and the fast switching component dominates the decay $(>80 \%)$.

\section{Conclusion}

In conclusion, we showed that fluorescent photochromic DAE possess a high potential for super-resolved imaging of apolar soft matter structures. Their properties can be tuned via facile synthesis of different derivatives, in particular for optimizing their photoswitching kinetics for different types of super-resolution methods, such as PALM (with or without UV activation), SOFI, and RESOLFT. The appropriate technique has to be chosen according to the research questions to be addressed, and according to the required temporal and spatial resolution. However, equally important, a suitable photoswitch for the corresponding technique is essential to obtain good super-resolved image. We demonstrated the application of different derivatives of diarylethene photoswitches to super-resolution fluorescence imaging, and pointed out ways to choose the "best" derivative based on a thorough analysis of the most important parameters such as switching kinetics, photobleaching, and fatigue. The outstanding performance of diarylethene photoswitches for PALM, SOFI and RESOLFT was shown comparatively on selfassembled block copolymer nanostructures. With our contribution, we hope to provide researchers the necessary tool to visualize polymer nanostructures and other apolar nanoscale compartments in materials and life science with nanoscopic resolution in situ and operando.

\section{Experimental Section}

Photophysical Properties of the Photoswitches: Absorption spectra were recorded on a JASCO V-550 UV-vis spectrometer, and emission spectra on a JASCO FP-8200 spectrofluorometer. For all measurements, spectroscopic grade solvents were used. Fluorescence lifetime measurements were performed with time-correlated single-photon counting on a MicroTime 200 Time-Resolved Confocal Fluorescence Microscope (MT200, PicoQuant $\mathrm{GmbH}$ ). Fluorescence quantum yields were determined in different solvents on a Hamamatsu Absolute PL Quantum Yield Measurement System C9920-02 employing an Ulbricht sphere according to the procedure reported by Würth et al. ${ }^{[63]}$ UV irradiation was carried out using a UV lamp (LUMOS 43, Atlas Photonics) at $366 \mathrm{~nm}$, and visible irradiation was performed with a $488 \mathrm{~nm}$ diode-pumped solid state laser $(200 \mathrm{~mW}$, Cobolt) and a $494 \mathrm{~nm}$ light-emitting diode (LED, $150 \mathrm{~mW}$, Avonec), respectively. The light-intensities $I_{0}$ were determined using the procedure described by Gauglitz (1 Einstein corresponds to 1 mol of photons). ${ }^{[64]}$ The fluorescence spectra of the compounds $1 c-10 \mathrm{c}$ are shown in Figure 1 and Figure S1 in the Supporting Information. The photophysical properties of the open and the closed form of the photoswitches are summarized in Table S1 in the Supporting Information.

PS- $b-P E O$ and PCL- $b$-PEO Cylindrical Micelles Preparation and Labeling: According to a procedure previously reported, ${ }^{[28,65]}$ a $10 \mathrm{mg} \mathrm{mL}^{-1}$ solution of the amphiphilic block copolymers PS- $b$-PEO $\left(M_{n, P S}=38 \mathrm{k}\right.$, $M_{n, P E O}=11 \mathrm{k}$, Polymer Source Inc. $)$ or PCL- $b$-PEO $\left(M_{n, P C L}=10 \mathrm{k}, M_{n, P E O}=\right.$ $5 \mathrm{k}$, Polymer Source Inc.), respectively, was prepared by dissolving the block copolymer in $0.1 \mathrm{~mL}$ of $\mathrm{a} \approx 10^{-4} \mathrm{M}$ solution of the photoswitch in chloroform. After the block copolymer had completely dissolved, $1 \mathrm{~mL}$ of an aqueous solution of sodium dodecyl sulfate (SDS, $1.0 \mathrm{mg} \mathrm{mL}^{-1}$ ) was added. While stirring, the chloroform slowly evaporated during $10 \mathrm{~h}$. The obtained solution was centrifuged at $5000 \mathrm{rpm}$ for $20 \mathrm{~min}$. The precipitate was dried under nitrogen atmosphere and dispersed in water as cylindrical micelles.

Fluorescence Microscopy: For PALM imaging, a $488 \mathrm{~nm}$ diode-pumped solid state laser (Cobolt Jive, $200 \mathrm{~mW}$ ) was used. Photoactivation of the dye molecules was achieved with a $375 \mathrm{~nm}$ diode laser (Toptica, $75 \mathrm{~mW}$ ). The combined laser beams were focused with a lens onto the back focal plane of a 100×/1.49 NA oil immersion objective (UAPON100×OTIRF, Olympus). The lens in front of the microscope was mounted onto a micrometer translation stage (Thorlabs) to enable TIRF illumination of the sample by moving the laser beam parallel to the microscope.

To perform widefield imaging under critical illumination, the laser was coupled into a multimode fiber (NA $0.22 \pm 0.02$, UM22-600 Thorlabs, $0.6 \mathrm{~mm}$ core diameter) which was shaken to average out the coherence and suppress interference effects to obtain homogeneous sample illumination. The end of the fiber was imaged onto $a \approx 40 \times 40 \mu \mathrm{m}^{2}$ 
area of the sample with a $100 \times / 1.49$ NA oil immersion objective (UAPON100×OTIRF, Olympus).

The samples were placed onto a xyz-piezo table (P-545.3R7, Physik Instrumente), mounted onto a commercial microscope (Olympus IX83). The fluorescence was collected using the same objective and was spectrally separated from the excitation laser light by a quad-line beamsplitter (zt405/488/561/640rpc, AHF Analysentechnik Tübingen), further magnified by two lenses $\left(f_{1}=200 \mathrm{~mm} \mathrm{AC254-200-A}\right.$ and $f_{2}=$ $400 \mathrm{~mm}$ AC254-400-A, both Thorlabs) and imaged onto the chip of an electron multiplying charge-coupled device (EMCCD) camera (Andor iXON Ultra 897). To suppress background stemming from the excitation laser, a longpass filter (ET500lp, AHF Analysentechnik) was placed between the two lenses, and a bandpass cleanup filter (Brightline HC $488 / 10$, AHF Analysentechnik) was placed in front of the laser. The total magnification was 200 -fold, resulting in an image with $80 \mathrm{~nm}$ per pixe of the CCD camera. All measurements were performed at $294 \mathrm{~K}$. The obtained movies were analyzed to reconstruct super-resolution images with the ThunderSTORM plugin for Image]. ${ }^{[5]}$

RESOLFT microscopy was carried out using a custom-built setup. RESOLFT requires three separate controlled light sources for activation, offswitching and read-out, respectively. Diode lasers from Cobolt $A B$ (Cobolt AB 06-MLD, 405, 488, and $488 \mathrm{~nm}$, Solna, Sweden) were chosen due to the possibility to directly modulate them, thus eliminating the need for an acousto-optic modulator or other light modulators. To generate the donutshaped light needed in the off-switching a vortex phase plate VPP-1 (RPC Photonics, NY, USA) was used. The three laser beams were combined and a TILL Yanus IV digital scan head, (Till Photonics, Graefelfing, Germany), was used for the $x y$-scanning. The system was built around a Leica DMI 6000 microscope, with a $100 \times$ STED $_{\text {white }}$ oil objective lens with 1.4 NA (Leica, Wetzlar, Germany). The stage was a custom-built stage mounted directly onto the objective lens to minimize any drift or vibration, with a built-in piezo for z-positioning (PI, Karlsruhe, Germany). The emission light was separated from the laser light using a longpass filter (ZT488rdc, Chroma, VT, USA) and further passed a bandpass filter (E535/70M, Chroma, VT, USA) before being collected by a SPAD (MPD, Bolzano, Italy). ImSpector was used to control scanners and detector. It sends a start signal to a pulse generator (Quantum Composers, CA, USA) to generate the trigger signal for the three laser. The lasers are switched on sequentially for each single pixel, and furthermore the detector is gated to ensure that only the emission light from the read-out phase is collected. For a more detailed description and explanation about RESOLFT see Testa and co-workers. ${ }^{[21]}$

For SOFI, a commercial VisiScope Spinning-Disk-DC Confocal System (Visitron Systems $\mathrm{GmbH}$ ) was used. Samples were placed onto a xyz-piezo table (P-545.3R7, Physik Instrumente), mounted onto a commercial inverted microscope (Nikon Eclipse Ti-E). Fluorescence was collected with a 100×/1.40 NA oil immersion objective (HP PLAN, Nikon) and was spectrally separated from the excitation laser light by a quad line beamsplitter (Di01-T405/488/568/647, Semrock) and then imaged onto the chip of an EMCCD camera (Andor iXON Ultra 888). To suppress background light originating from the excitation laser, a bandpass filter (ET525/50m, Chroma) was placed in the emission path. The total magnification was 180, resulting in an image with $72 \mathrm{~nm}$ per pixel of the CCD camera. All measurements were performed at $294 \mathrm{~K}$. The obtained movies were analyzed to reconstruct super-resolution images according to the procedure described below.

\section{Acknowledgements}

This work was supported by the German Research Foundation (DFG) within project A6 of the SFB 985 "Functional Microgels and Microgel
Systems" and by the Research Grant HU1682/3-1. Moreover, the financial support from the RWTH competence center FLAMENCO within the profile area Molecular Science and Engineering (MSE) is appreciated. I.T. acknowledges her ERC starting grant 'MoNaLISA' funding (http://dx.doi.org/10.13039/501100000781) for supporting the project. J.D. was funded by the Carlsberg Foundation. The authors thank J.M. Hoffmann for his assistance in fluorescence lifetimes measurements and J.R. Perez for fluorescence quantum yields measurements.

\section{Conflict of Interest}

The authors declare no conflict of interest.

\section{Keywords}

block copolymers, diarylethenes, nanostructures, photoswitches, superresolution fluorescence microscopy

[1] A. H. Gröschel, A. Walther, T. I. Löbling, F. H. Schacher, H. Schmalz, A. H. E. Müller, Nature 2013, 503, 247

[2] K. Matyjaszewski, Science 2011, 333, 1104

[3] M. Kather, M. Skischus, P. Kandt, A. Pich, G. Conrads, S. Neuss, Angew. Chem., Int. Ed. 2017, 56, 2497.

[4] R. M. Choueiri, E. Galati, H. Thérien-Aubin, A. Klinkova, E. M. Larin, A. Querejeta-Fernández, L. Han, H. L. Xin, O. Gang, E. B. Zhulina, M. Rubinstein, E. Kumacheva, Nature 2016, 538, 79.

[5] J. Lee, K. Baek, M. Kim, G. Yun, Y. H. Ko, N.-S. Lee, I. Hwang, J. Kim, R. Natarajan, C. G. Park, W. Sung, K. Kim, Nat. Chem. 2014, 6, 97.

[6] M. T. Proetto, A. M. Rush, M.-P. Chien, P. Abellan Baeza, J. P. Patterson, M. P. Thompson, N. H. Olson, C. E. Moore, A. L. Rheingold, C. Andolina, J. Millstone, S. B. Howell, N. D. Browning, J. E. Evans, N. C. Gianneschi, J. Am. Chem. Soc. 2014, 136, 1162.

[7] E. T. Herruzo, A. P. Perrino, R. Garcia, Nat. Commun. 2014, 5, 3126.

[8] S. W. Hell, Science 2007, 316, 1153.

[9] S. W. Hell, S. Jakobs, L. Kastrup, Appl. Phys. A: Mater. Sci. Process. 2003, 77, 859.

[10] M. Heilemann, S. van de Linde, M. Schüttpelz, R. Kasper, B. Seefeldt, A. Mukherjee, P. Tinnefeld, M. Sauer, Angew. Chem., Int. Ed. 2008, 47, 6172.

[11] B. Huang, W. Wang, M. Bates, X. Zhuang, Science 2008, 319, 810.

[12] E. Betzig, G. H. Patterson, R. Sougrat, O. W. Lindwasser, S. Olenych, J. S. Bonifacino, M. W. Davidson, J. Lippincott-Schwartz, H. F. Hess, Science 2006, 313, 1642.

[13] T. Dertinger, R. Colyer, G. lyer, S. Weiss, J. Enderlein, Proc. Natl. Acad. Sci. USA 2009, 106, 22287.

[14] H. Deschout, T. Lukes, A. Sharipov, D. Szlag, L. Feletti, W. Vandenberg, P. Dedecker, J. Hofkens, M. Leutenegger, T. Lasser, A. Radenovic, Nat. Commun. 2016, 7, 13693.

[15] J. B. Grimm, T. Klein, B. G. Kopek, G. Shtengel, H. F. Hess, M. Sauer, L. D. Lavis, Angew. Chem., Int. Ed. 2016, 55, 1723.

[16] G. T. Dempsey, J. C. Vaughan, K. H. Chen, M. Bates, X. Zhuang, Nat. Methods 2011, 8, 1027.

[17] F. Balzarotti, Y. Eilers, K. C. Gwosch, A. H. Gynnå, V. Westphal, F. D. Stefani, J. Elf, S. W. Hell, Science 2016, 355, 606. 
[18] W. R. Legant, L. Shao, J. B. Grimm, T. A. Brown, D. E. Milkie, B. B. Avants, L. D. Lavis, E. Betzig, Nat. Methods 2016, 13, 359.

[19] I. Testa, E. D'Este, N. T. Urban, F. Balzarotti, S. W. Hell, Nano Lett. 2015, 15, 103.

[20] T. Grotjohann, I. Testa, M. Reuss, T. Brakemann, C. Eggeling, S. W. Hell, S. Jakobs, eLife 2012, 1, e00248.

[21] T. Grotjohann, I. Testa, M. Leutenegger, H. Bock, N. T. Urban, F. Lavoie-Cardinal, K. I. Willig, C. Eggeling, S. Jakobs, S. W. Hell, Nature 2011, 478, 204.

[22] J. Kwon, J. Hwang, J. Park, G. R. Han, K. Y. Han, S. K. Kim, Sci. Rep. 2015, 5, 17804 .

[23] B. Roubinet, M. L. Bossi, P. Alt, M. Leutenegger, H. Shojaei, S. Schnorrenberg, S. Nizamov, M. Irie, V. N. Belov, S. W. Hell, Angew. Chem., Int. Ed. 2016, 55, 15429.

[24] A. M. Chizhik, S. Stein, M. O. Dekaliuk, C. Battle, W. Li, A. Huss, M. Platen, I. A. T. Schaap, I. Gregor, A. P. Demchenko, C. F. Schmidt, J. Enderlein, A. I. Chizhik, Nano Lett. 2016, 16, 237.

[25] D. Wöll, C. Flors, Small Methods 2017, 1, 1700191.

[26] O. Nevskyi, D. Sysoiev, A. Oppermann, T. Huhn, D. Wöll, Angew. Chem., Int. Ed. 2016, 55, 12698.

[27] A. P. H. Gelissen, A. Oppermann, T. Caumanns, P. Hebbeker, S. K. Turnhoff, R. Tiwari, S. Eisold, U. Simon, Y. Lu, J. Mayer, W. Richtering, A. Walther, D. Wöll, Nano Lett. 2016, 16, 7295.

[28] J. Yan, L.-X. Zhao, C. Li, Z. Hu, G.-F. Zhang, Z.-Q. Chen, T. Chen, Z.-L. Huang, J. Zhu, M.-Q. Zhu, J. Am. Chem. Soc. 2015, 137, 2436.

[29] A. Aloi, A. Vargas Jentzsch, N. Vilanova, L. Albertazzi, E. W. Meijer, I. K. Voets, J. Am. Chem. Soc. 2016, 138, 2953.

[30] C. E. Boott, R. F. Laine, P. Mahou, J. R. Finnegan, E. M. Leitao, S. E. D. Webb, C. F. Kaminski, I. Manners, Chem. Eur. J. 2015, 21, 18539.

[31] M. W. Gramlich, J. Bae, R. C. Hayward, J. L. Ross, Opt. Express 2014, 22,8438 .

[32] C. Li, H. Yan, L.-X. Zhao, G.-F. Zhang, Z. Hu, Z.-L. Huang, M.-Q. Zhu, Nat. Commun. 2014, 5, 5709.

[33] C. E. Oneil, J. M. Jackson, S.-H. Shim, S. A. Soper, Anal. Chem. 2016, 88, 3686

[34] S. Onogi, H. Shigemitsu, T. Yoshii, T. Tanida, M. Ikeda, R. Kubota, I. Hamachi, Nat. Chem. 2016, 8, 743.

[35] A. Kaltbeitzel, K. Friedemann, A. Turshatov, C. Schönecker, I. Lieberwirth, K. Landfester, D. Crespy, Macromol. Chem. Phys. 2017, 218, 1600547.

[36] M. Irie, T. Fukaminato, K. Matsuda, S. Kobatake, Chem. Rev. 2014, $114,12174$.

[37] V. Valderrey, A. Bonasera, S. Fredrich, S. Hecht, Angew. Chem., Int. Ed. 2017, 56, 1914

[38] T. Leydecker, M. Herder, E. Pavlica, G. Bratina, S. Hecht, E. Orgiu, P. Samorì, Nat. Nanotechnol. 2016, 11, 769.

[39] A. Fuhrmann, R. Göstl, R. Wendt, J. Kötteritzsch, M. D. Hager, U. S. Schubert, K. Brademann-Jock, A. F. Thünemann, U. Nöchel, M. Behl, S. Hecht, Nat. Commun. 2016, 7, 13623.
[40] B. Roubinet, M. Weber, H. Shojaei, M. Bates, M. L. Bossi, V. N. Belov, M. Irie, S. W. Hell, J. Am. Chem. Soc. 2017, 139, 6611.

[41] Y. Arai, S. Ito, H. Fujita, Y. Yoneda, T. Kaji, S. Takei, R. Kashihara, M. Morimoto, M. Irie, H. Miyasaka, Chem. Commun. 2017, 53, 4066.

[42] T. Weil, T. Vosch, J. Hofkens, K. Peneva, K. Müllen, Angew. Chem., Int. Ed. 2010, 49, 9068.

[43] K. Uno, H. Niikura, M. Morimoto, Y. Ishibashi, H. Miyasaka, M. Irie, J. Am. Chem. Soc. 2011, 133, 13558.

[44] K. I. Mortensen, L. S. Churchman, J. A. Spudich, H. Flyvbjerg, Nat. Methods 2010, 7, 377.

[45] M. Herder, B. M. Schmidt, L. Grubert, M. Patzel, J. Schwarz, S. Hecht, J. Am. Chem. Soc. 2015, 137, 2738.

[46] M. Herder, F. Eisenreich, A. Bonasera, A. Grafl, L. Grubert, M. Pätzel, J. Schwarz, S. Hecht, Chem. Eur. J. 2017, 23, 3743.

[47] M. Irie, K. Sakemura, M. Okinaka, K. Uchida, J. Org. Chem. 1995, 60, 8305 .

[48] D. Bléger, S. Hecht, Angew. Chem., Int. Ed. 2015, 54, 11338.

[49] J. R. Siekierzycka, C. Hippius, F. Würthner, R. M. Williams, A. M. Brouwer, J. Am. Chem. Soc. 2010, 132, 1240.

[50] J. R. Siekierzycka, C. Hippius, F. Würthner, R. M. Williams, A. M. Brouwer, Cent. Eur. J. Chem. 2014, 12, 937.

[51] T. Fukaminato, T. Umemoto, Y. Iwata, S. Yokojima, M. Yoneyama, S. Nakamura, M. Irie, J. Am. Chem. Soc. 2007, 129, 5932.

[52] D. Wöll, E. Braeken, A. Deres, F. C. De Schryver, H. Uji-i, J. Hofkens, Chem. Soc. Rev. 2009, 38, 313.

[53] B. M. I. Flier, M. C. Baier, J. Huber, K. Müllen, S. Mecking, A. Zumbusch, D. Wöll, J. Am. Chem. Soc. 2012, 134, 480.

[54] K. Paeng, L. J. Kaufman, Macromolecules 2016, 49, 2876.

[55] S. Adhikari, M. Selmke, F. Cichos, Phys. Chem. Chem. Phys. 2011, 13, 1849.

[56] A. Deres, G. A. Floudas, K. Müllen, M. Van der Auweraer, F. De Schryver, J. Enderlein, H. Uji-i, J. Hofkens, Macromolecules 2011, 44, 9703

[57] M. Ovesný, P. Kř́žžek, J. Borkovec, Z. Švindrych, G. M. Hagen, Bioinformatics 2014, 30, 2389.

[58] R. P. J. Nieuwenhuizen, K. A. Lidke, M. Bates, D. L. Puig, D. Grünwald, S. Stallinga, B. Rieger, Nat. Methods 2013, 10, 557.

[59] M. Lakadamyali, H. Babcock, M. Bates, X. Zhuang, J. Lichtman, PLoS One 2012, 7, e30826.

[60] A. Blanazs, J. Madsen, G. Battaglia, A. J. Ryan, S. P. Armes, J. Am. Chem. Soc. 2011, 133, 16581.

[61] S. C. Stein, A. Huss, D. Hähnel, I. Gregor, J. Enderlein, Opt. Express 2015, 23, 16154.

[62] I. Testa, N. T. Urban, S. Jakobs, C. Eggeling, K. I. Willig, S. W. Hell, Neuron 2012, 75, 992.

[63] C. Würth, M. Grabolle, J. Pauli, M. Spieles, U. Resch-Genger, Nat. Protocols 2013, 8, 1535.

[64] G. Gauglitz, S. Hubig, J. Photochem. 1985, 30, 121.

[65] J. Zhu, R. C. Hayward, J. Am. Chem. Soc. 2008, 130, 7496. 